\title{
Performance Analysis for Perfect Difference Network and N Complete Network using NS-2
}

\author{
Jyotsana Nandagaoli \\ Department of Computer, \\ Mumbai University, \\ Pillai Institute of Information Technology, \\ New Panvel
}

\begin{abstract}
These paper is based on simulation of the Perfect Difference Network (PDN) and N-Complete Network $\left(\mathrm{K}_{\mathrm{n}}\right)$ using NS2 (Network simulator) for 7 and 13nodes. This paper is not only doing simulation part and also the analysis sections to find out which topology is better with respect to cost and performance. This paper is comparing the performance of PDN architecture and mesh architecture in terms of various parameters thus explaining the benefits of replacing mesh architecture by PDN architecture. Throughput, packet loss are the primary parameters for comparison while change in traffic rates, link failure etc. are the secondary parameters. In some conditions, mesh provides better performance than PDN but it is compensated by reduced cost (as the number of link is reduced) by PDN.
\end{abstract}

\section{General Terms}

Throughput; Packet Loss

\section{Keywords}

Perfect Difference Network; N Complete Network; PDS; ns-2

\section{INTRODUCTION}

Network is a set of devices connected by link for communication. The decision of the interconnection network may affect several characteristics of the final system, including implementation cost, performance, reliability, and scalability [1]. On the basis of characteristic, network is classified in the simplest ring, linear architecture, star architecture, stronger hypercube, pancake and complete network architectures. Perfect Difference Network was introduced in [1] as a hyperstar later name was changed. Perfect Difference Network (PDN) was introduced has 2 diameters, so one node is connected to another node in one or two hope [2]. Perfect Difference Network (PDN) has better performance with lesser cost. The $\mathrm{N}$ complete (Kn) architecture consists of $\mathrm{n}$ nodes, with each node linked to every other node. This architecture has a total communication bandwidth of $n(n-1) / 2$ and $n(n-1) / 2$ links. $N$ complete $(K n)$ Network planning is hard to give, because of both the high cost of nodes with numerous correspondence channels and absence of adaptability for framework development. In any case, it is a perfect construction modeling against which the correspondence execution of different architectures can be compared. The design goals for this paper can be described as follows:

\section{Platform based design}

2. Minimization in cost and area.

3. Compare between complete network and perfect difference network

\author{
Jagdish W. Bakal, PhD \\ Phd, Principal, \\ Mumbai University, \\ Shivajirao S Jondhale College of Engineering, \\ Dombivali(E)
}

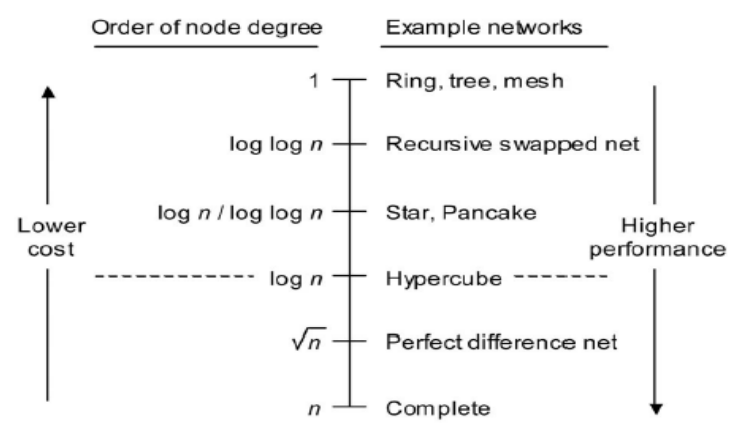

Figure 1: The spectrum of networks in terms of node degree. The hypercube, with its excellent performance and logarithmic diameter, is often used as a reference point for comparison [2].

\section{N COMPLETE NETWORK AND PERFECT DIFFERENCE NETWORK}

Optimizing links in interconnections network is extremely vital for contemporary computer, electronic, and communication systems. The most popular architecture i.e. $\mathrm{N}$ complete Network (Full Mesh) in which each node is link to every other node. An implementation of this architecture can't be possible in practically because size of network increased with respect to increase interconnecting link between nodes. New architecture introduces Perfect Difference Network (PDN), it is an asymptotically optimal method for connecting a set of nodes into a Perfect Difference Network (PDN) with diameter 2, and any node is connected to any other node in 1 or 2 hops. Routing performance of PDN is almost same to $\mathrm{N}$ Complete Network. Cost Implementation of PDN is low as compared to N Complete Network.

\subsection{Perfect Difference Set}

Prefect Difference set is mathematical tool for calculating optimizing number node in asymptotic manner for perfect difference Network [2].Perfect difference sets were first Discussed by J. Singer in 1938 in terms of points and lines in a finite projective planes [3].

Perfect Difference Set (PDS) - A set $\{s 0, s 1, \ldots \ldots . ., s \delta\}$ of $\delta$ +1 integers having the property that their $\delta 2+\delta$ differences $s i-s j, 0 \leq i \neq j \leq \delta$, are congruent, modulo $(\delta 2+\delta+1)$, to the integers $1,2, \ldots, \delta 2+\delta$ in some order is a perfect difference set of order $\delta$. Perfect difference sets are sometimes called simple difference sets. Perfect Difference sets with order $\delta$ as a power of prime number and number of nodes, $n=\delta^{2}+\delta+1$. PDS need not contain an integer outside the interval $\left[0, \delta^{2}+\right.$ $\delta]$, because any integer outside the interval can be replaced by another integer in the interval without affecting the defining property of the PDS [3].Perfect Difference Set $\left\{s_{0}, s_{1}, \ldots\right.$, $\left.s_{\delta}\right\}$ is reduced to normal PDS if it contains the integers 0 and 
1. A reduced PDS is in normal form if it satisfies $s i<s i+1 \leq \delta 2$ $+\delta, 0 \leq i<\delta$.

\subsection{Perfect difference Network (PDN)}

Perfect difference network is depended on the mathematical notion of perfect difference set. Consider the normal-form PDS $\{0,1, \mathrm{~s} 2, \ldots, \mathrm{s} \delta\}$ of order $\delta$. We can construct a direct interconnection network with $\mathrm{n}=\delta 2+\delta+1$ nodes based on this PDS.

Definition 1: Perfect difference network (PDN) based on the PDS $\{0,1, \mathrm{~s} 2, \ldots, \mathrm{s} \delta\}-$ There are $\mathrm{n}=\delta 2+\delta+1$ nodes, numbered 0 to $\mathrm{n}-1$. Node $\mathrm{i}$ is connected via directed links to nodes $\mathrm{i} \pm \square 1$ and $\mathrm{i} \pm \square \operatorname{si}(\bmod n)$, for $2 \delta \square \mathrm{i}<\leq \square$. Because all index expressions in this paper are evaluated modulo $n$, henceforth we will delete the qualifier "mod n." The preceding connectivity leads to a chordal ring of in- and outdegree $d=2 \leq \square$ and diameter $D=2$. Because for each link from node $i$ to node $\mathrm{j}$, the reverse link $(\mathrm{j}, \mathrm{i})$ also exists, the network corresponds to an undirected graph. Fig 2 and fig. 3 shows graphical representation of Perfect Difference Network of $\delta=2,3$.

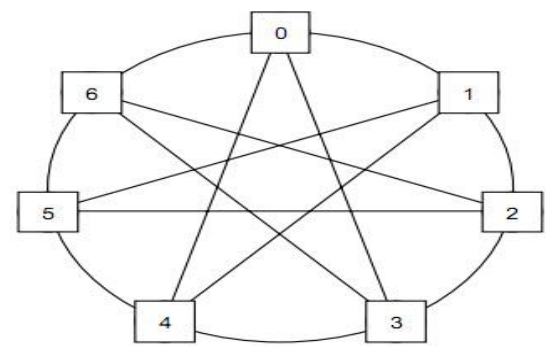

Figure2: PDN with $\mathbf{n}=7$ nodes based on the perfect difference set $\{0,1,3\}$

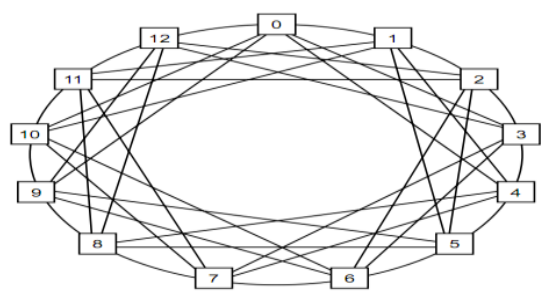

Figure 3: PDN with $n=13$ nodes based on the perfect difference set $\{0,1,3,9\}$

Table 1 shows examples of the Perfect Difference Set for Several order of sets.

\begin{tabular}{|ccc|}
\hline $\mathrm{n}$ & $\mathrm{N}$ & PDS \\
\hline 2 & 7 & $\{0,1,3\}$ \\
\hline 3 & 13 & $\{0,1,3,9\}$ \\
\hline 3 & 13 & $\{0,1,4,6\}$ \\
\hline 4 & 21 & $\{0,1,4,14,16\}$ \\
\hline 5 & 31 & $\{0,1,4,1,6,13,21\}$ \\
\hline 5 & 31 & $\{0,1,8,11,13,17\}$ \\
\hline 5 & 31 & $\{0,1,3,13,32,36,43,52\}$ \\
\hline 7 & 57 &
\end{tabular}

\section{N COMPLETE NETWORK}

The $\mathrm{N}$ complete $(\mathrm{Kn})$ architecture consists of $\mathrm{n}$ nodes, with each node connected to all node of the architecture. This network has $\mathrm{n}(\mathrm{n}-1) / 2$ links and a total communication bandwidth of $n(n-1) / 2$, assuming unit capacity links. The $N$ complete $(\mathrm{Kn})$ Network has diameter $\mathrm{D}=1$. This is because direct communication performed in nodes, there is no required intermediate switch. Physically, $\mathrm{N}$ complete $(\mathrm{Kn})$ architecture is hard to implement, cost of nodes is high with respect to increase communication links and lack of scalability for system growth. However, Communication Performance of this architecture is best as compared to other architectures. $\mathrm{N}$ complete Network is in view of a full diagram outline in which every hub is associated with each other hub. This kind of system is not adaptable when system size expands and cost likewise high. PDNs have a diameter of two and a node degree of roughly 2 , which put them near to $\mathrm{N}$ Complete Network in terms of routing performance and much lower regarding implementation cost.

\section{SIMULATION ENVIRONMENT}

NS is an object oriented discrete event simulator. Simulator maintains list of events and executes one event after another Single thread of control where no locking or race condition is present. Back end used in NS-2 is C++ event scheduler which is used in protocols mostly. Again it's very fast to run, and provides more control Front end used in NS-2 is OTCL which creates scenarios, and extensions to $\mathrm{C}++$ protocols [6]. It is easier to write and change. This paper compare throughput and No Packet of Packet Loss of PDN and N Complete Network for 7 nodes and 13 nodes. First we create two different Networks using Network Simulation version 2.

\section{EXPERIMENTAL RESULT}

This paper compares performance (in term of throughput and packet loss) of PDN and N Complete Network for 7 Node and 13 nodes. Table 2 shows communication parameter for Perfect Difference Network and N Complete Network

.Table 2 Shows Communication Parameter

\begin{tabular}{|l|l|}
\hline Nodes & 7 and 13 \\
\hline Topologies & PDN and N complete \\
\hline Traffic Rate & FTP \\
\hline Network Protocol & TCP \\
\hline Network Parameter & Throughput, Packet Loss \\
\hline Bandwidth & 1 MBPS \\
\hline Packet Size & 500 bytes \\
\hline Routing Protocol & Distance Vector \\
\hline Queue Management & Drop Trail \\
\hline Mechanism & Node 0 \\
\hline Source Node & Node 5 \\
\hline Destination Node & Dynamic \\
\hline Routing Strategic & \\
\hline
\end{tabular}

\subsection{Throughput result for 7 nodes}

Throughput for PDN $n=7$ is analyzed based on PDS $\{0,1,3\}$ and N Complete Network. As observed in Fig 4 and Fig 5 throughput of the PDN is good as compare to $\mathrm{N}$ Complete Network in the case of links fail.As see in Fig 5, 5 
links are failed respective networks throughput performance of PDN is more.

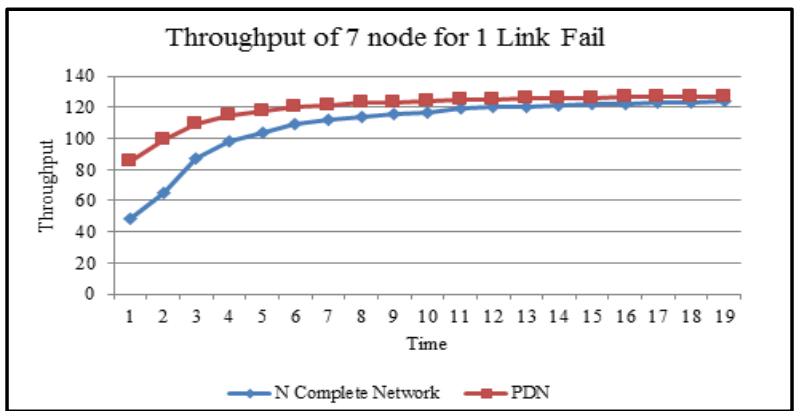

Figure 4: Shows Graphical representation of throughput for 7 nodes of 1 link Fail

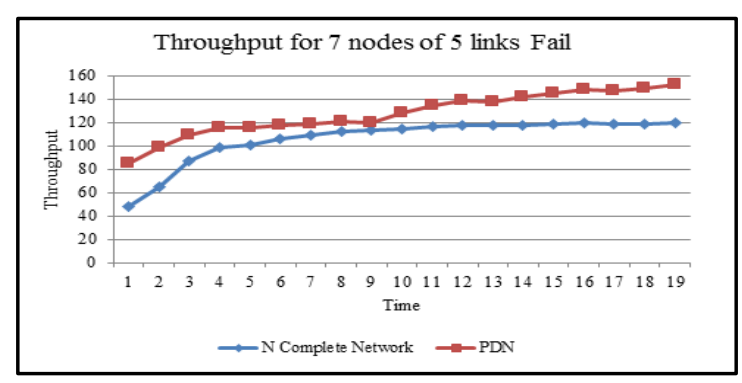

Figure 5: Shows Graphical representation of throughput for 7 Node of 5 links Fail.

\subsection{Throughput result for 13 Nodes}

As observed in fig 6 throughput for PND $n=13$ based on $\operatorname{PSD}\{0,1,4$,$\} and \mathrm{N}$ complete network is steady for 1 link fail. In fig 7, throughput for $\mathrm{N}$ Complete Network and PDN varies according to links fail. Throughput for N Complete Network is degraded when more links fail as compared to PDN.

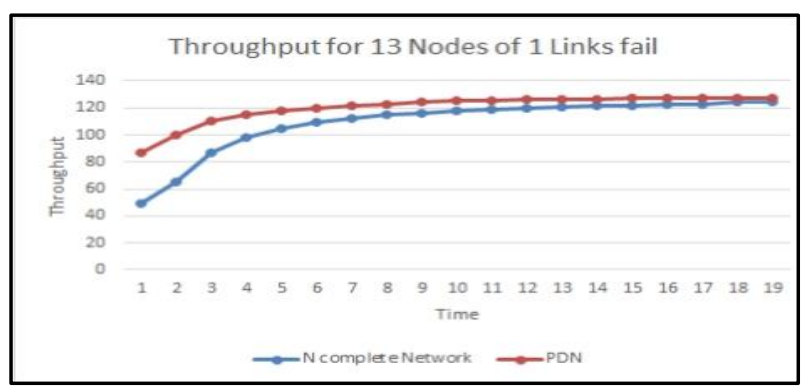

Figure 6: Shows Graphical representation of Throughput for 13 Nodes of 1 Link fail

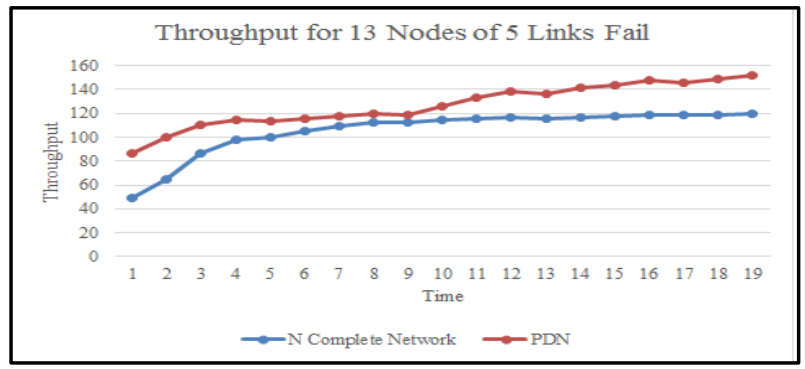

Figure 7: Shows Graphical representation of Throughput for 13 Nodes of 5 Link fail

\subsection{Packet Loss}

A percentage of packet loss in N Complete Network is more compare to Perfect Difference Network with respect to increase no of links fails as observed in table 3 . The Percentage of packet Loss for 1 link fail of PDN 7 and 13 nodes is almost negligible i.e. $0.03 \%$.

Table 3 Shows No of Packet Loss for PDN and N Complete Network

\begin{tabular}{|c|c|c|c|c|}
\hline \multicolumn{4}{|c|}{ Percentages of Packet Loss } \\
\hline & \multicolumn{2}{|c|}{ 7 Nodes } & \multicolumn{2}{c|}{ 13 Nodes } \\
\cline { 2 - 5 } & $\begin{array}{c}\text { 1 Link } \\
\text { Fail }\end{array}$ & $\begin{array}{c}\text { 5 Links } \\
\text { Fail }\end{array}$ & $\begin{array}{c}\text { 1 Link } \\
\text { Fail }\end{array}$ & $\begin{array}{c}\text { 5 Links } \\
\text { Fail }\end{array}$ \\
\hline $\begin{array}{c}\mathbf{N} \\
\text { Complete } \\
\text { Network }\end{array}$ & $0.11 \%$ & $0.24 \%$ & $0.10 \%$ & $0.27 \%$ \\
\hline PDN & $0.03 \%$ & $0.12 \%$ & $0.03 \%$ & $0.18 \%$ \\
\hline
\end{tabular}

\section{CONCLUSION}

The Perfect Difference Network $\mathrm{n}=7$ and 13 based on PDS $\{0,1,3\}$ and $\{0,1,4,9\}$ and $N$ Complete Network with nodes 7 \& 13 is analyzed and simulated. For simulation of network has used NS2 as it is an open source discrete event simulator and it again provides one to one correspondence between a class in compiled hierarchy and the one in interpreted hierarchy. This paper has tested the network for protocols TCP. Again showed details study of the network parameters like throughput, packet lost for the same Perfect Difference Network for values of $\delta=2,3$ and N Complete Network where we found throughput for the designed network.

On the basis of above result conclude that throughput of the $\mathrm{N}$ complete network is less as compare to PDN. More links fails throughput gets degrade in N Complete Network. This paper has analyzed percentage of packet loss in $\mathrm{N}$ Complete Network is more than Perfect Difference Network. If no links fails in N Complete Network increase, then no of packet loss increase compare to PDN. The advantage of PDN has low cost compare to the $\mathrm{N}$ Complete Network. Cost of PDN is less by reducing links.

\section{ACKNOWLEDGMENTS}

The Authors would like to thank Dr. Mikhail A. Rakov and Mr. Behrooz Parhami for give brief introduction of Perfect Difference Network. Special thanks to Dr. Mahendra Gaikwad BDCOE Wardha, for simulation suggestion and early feedback.

\section{REFERENCES}

[1] M. Rakov, "Method of Interconnecting Nodes and a Hyperstar Interconnection Structure," US Patent No. 5 734 580, Mar. 1998.

[2] BehroozParhami, Fellow, IEEE, and Mikhail Rakov, "Perfect Difference Networks and Related Interconnection Structures for Parallel and Distributed Systems," IEEE transactions on parallel and distributed systems, vol. 16, no. 8, august 2005, pp714-724

[3] J. Singer, "A Theorem in Finite Projective Geometry and Some Applications to Number Theory," Trans. Am. Math. Soc., vol. 43, pp. 377-385, 1938. 
[4] B.Parhami and M. Rakov, "Performance, Algorithmic, and Robustness Attributes of Perfect Difference Networks," IEEE.Trans. Parallel and Distributed Systems, vol. 16, no. 8, pp. 725-736, 2005.

[5] Mahendra Gaikwad, RajendraPatrikar, Abhay Gandhi "Latency Performance of Chordal Ring Perfect Difference Network Topology for Network on Chip Architecture using NS-2", International Journal of Computer Applications (0975 - 8887) Volume 34No.10, November 2011.
[6] Object oriented TCL script and NS Manual http://www.isi.edu/nsnam/ns/ns documentation.html

[7] B.W. Arden and H. Lee, "Analysis of Chordal Ring Networks," IEEE Trans. Computers, vol. 30, no. 4, pp. 291-295, Apr. 1981.

[8] Rakesh Kumar Katare, N.S. Chaudhari, Shazad Ahmed Mughal "Study of Link Utilization of Perfect Difference Network and Hypercube" 